\title{
Dissection of Herpesvirus Envelopment by Electron Microscopy at High Spatial and High Temporal Resolution
}

\author{
P. Wild*, C. Senn**, H. Leuzinger,* E. M. Schraner*, U. Ziegler,*** P. Walther,**** and M. \\ Müller***** \\ *Electron Microscopy, Institutes of Veterinary Anatomy and of **Virology, University of Zürich, \\ CH-8057 Zürich, Switzerland, ***Institute of Anatomy, University of Zürich, ****Electron \\ Microscopic Unit, University of Ulm, D-89069 Germany, *****Laboratory for Electron \\ Microscopy, Institute of Biochemistry, Swiss Federal Institute of Technology, CH-8092 Zürich
}

Capsids of herpesviruses bud through the inner nuclear membrane acquiring both the viral envelope and tegument. On the basis of slowly fixed material, which results in poor spatial and temporal resolution, the idea was born that the envelope derived from the inner nuclear membrane is inserted into the outer nuclear membrane by fusion releasing both capsid and tegument into the cytoplasm. The final envelope is speculated to originate at the trans Golgi network forming vacuoles. Their membranes wrap the capsid after tegument has been deposited [1]. However, fusion of the viral envelope with the outer nuclear membrane has never been shown so far. Instead it was shown that virions are transported from the perinuclear space into RER cisternae and membrane compartments devoid of ribosomes [2] ruling out fusion of the envelope with membranes of cellular compartments virions are transported through.

The idea of envelopment followed by de-envelopment and re-envelopment faces an uneconomical cellular process. This and the fact that membrane bound processes are difficult to capture in conventionally fixed material we investigated envelopment of bovine herpesvirus 1 and herpes simplex virus 1 by high resolution microscopy employing cryo-fixation followed by freezesubstitution or freeze etching, and by confocal microscopy. Examination of cells at various times post infection revealed that the process taking place at the outer nuclear membrane has nothing common with membrane fusion. It rather is identical to the process taking place at the inner nuclear membrane and is thus considered to represent budding capsids from the cytoplasm into the perinuclear space. Careful examination of the nuclear periphery revealed impaired nuclear pores through which capsids gain direct access to the cytoplasm. Nuclear pore complexes are probably disassembled and its components become distributed within the cytoplasm as revealed by confocal microscopy. The Golgi complex also showed two distinctly different processes of virus-membrane interaction. One takes place at lateral sides and is identical to the packaging process in the secretory pathway. The other process referred to as wrapping is identical to budding at nuclear membranes.

The results imply the existence of two diverse pathways. In the packaging pathway, capsids bud through the inner nuclear membrane into the perinuclear space acquiring tegument and envelope that contains a protein for preventing membrane fusion so that virions can be transported via RER cisternae directly into the Golgi complex [3] for packaging into transport vacuoles that deliver their content to the cell periphery. In the wrapping pathway, capsids are delivered to the cytoplasm via impaired nuclear pores [4]. Cytoplasmic capsids approach the outer nuclear membrane, membranes of RER and Golgi complex at any side where they induce budding. Budding of capsids at Golgi membranes of narrow cisternae leads to virions within concentric transport vacuoles that contain proteins considered likely to prevent fusion of the envelope with the vacuolar membrane. 

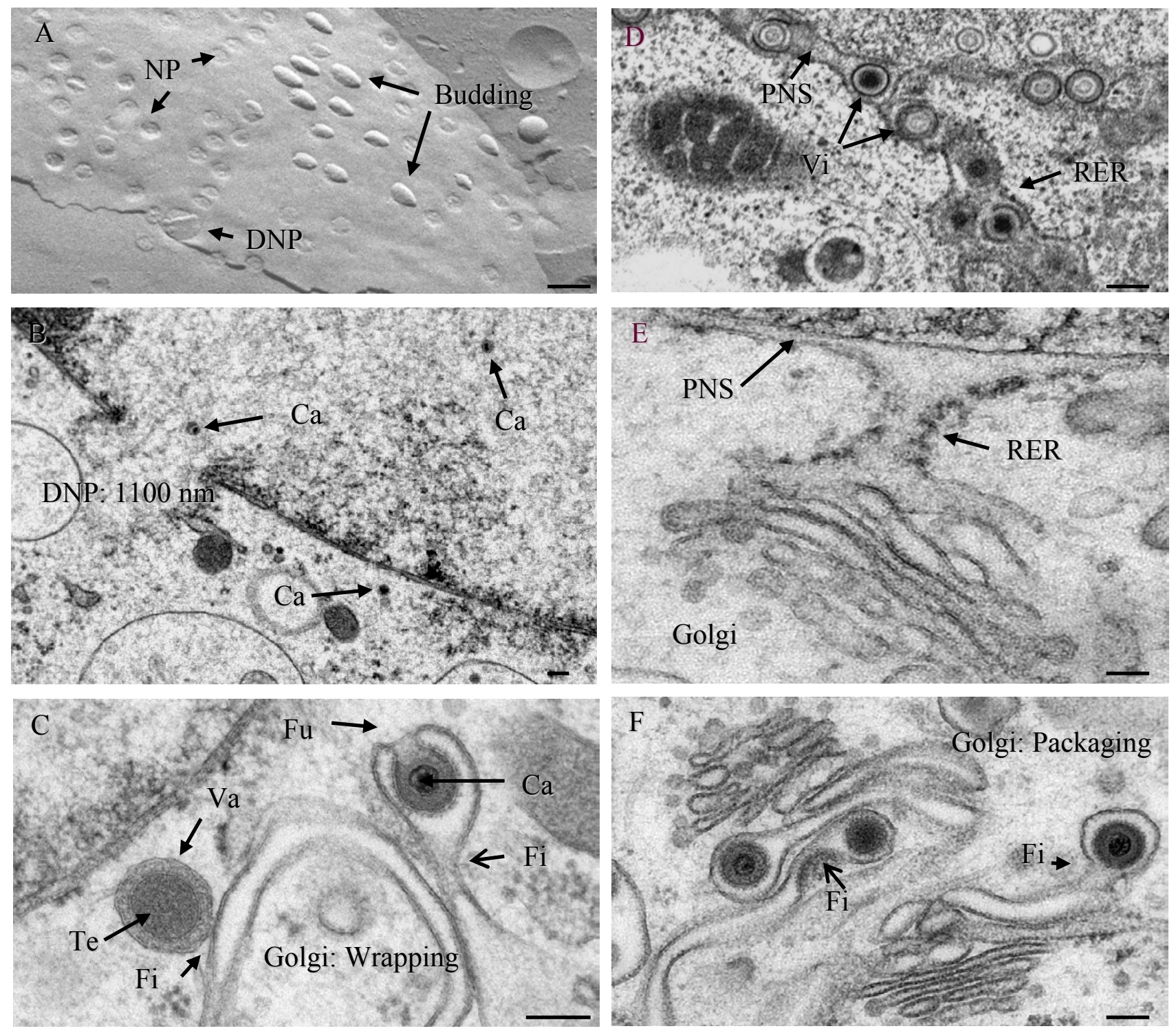

Fig.1. Wrapping pathway and packaging pathway of herpesvirus envelopment. In the wrapping pathway capsids (Ca) escape the nucleus via dilated nuclear pores (DNP; NP = intact nuclear pores), shown after freeze-fracture (A) and freeze-substitution (B), and bud at Golgi membranes (C). Fusion $(\mathrm{Fu})$ of Golgi membranes followed by fission $(\mathrm{Fi})$ results in a virion $(\mathrm{Ca}$ and Tegument $=\mathrm{Te}$, tangential section) within a vacuole ( $\mathrm{Va}$ - a process called wrapping. In the packaging pathway, capsids bud at the inner nuclear membrane into the perinuclear space (A, D), resulting in virions (Vi) that are transported via RER cisternae (D) into Golgi cisternae, and are packaged into vacuoles containing one (F) or more virions. The perinuclear space (PNS), RER cisternae and Golgi cisternae form a continuum (E). Bars $=200 \mathrm{~nm}$.

\section{References}

[1] H. Granzow et al., J. Virol. 75 (2001) 3675

[2] L.M. Stannard et al., Arch. Virol. 141 (1996) 505

[3] P. Wild et al., Micron 33 (2002) 327

[4] P. Wild et al., J. Virol. 79 (2005) 1071 